\title{
Gestión de la calidad en enfermería en un centro periférico de diálisis
}

\author{
Bernardo Manzano Salazar* - Carlos Vázquez Viejo* - Enrique J. Gutiérrez Guillén** - \\ Jorge Manuel Rivero Mesa*** - María Rivero Mesa****
}

*Diplomado en Enfermería

**Ingeniero Técnico Industrial

$* * *$ Licenciado en Derecho

****Licenciada en Administración de Empresas

Centro de hemodiálisis Montequinto. Dos Hermanas. Sevilla.

\section{Sr. Director:}

Es indudable la relevancia que ha supuesto la implantación de Sistemas de Gestión de la Calidad en el ámbito de la Enfermería Nefrológica. Las recomendaciones del modelo EFQM (European Foundation for Quality Management) y las Normas Internacionales de Estandarización (ISO) son de introducción reciente en el ámbito sanitario ${ }^{1}$ pero con repercusiones cada vez más importantes. Calidad es un término bastante genérico y que recibe numerosas definiciones según el contexto en el que se use .; Calidad según la Real Academia de la Lengua Española se define como: "Propiedad o conjunto de propiedades inherentes a una cosa que permiten apreciarla como igual, mejor o peor que las restantes de su especie" ${ }^{\prime \prime}$. La definición que del término Calidad da la norma UNE-EN ISO 9000 es la siguiente: "Grado en el que un conjunto de características inherentes cumple con los requisitos".

Aunque puede resultar dificultoso trasladar todo esto al ámbito de la Enfermería, nosotros entenderemos como Sistema de Gestión de la Calidad a todos aquellos elementos interrelacionados que imparten, administran

Correspondencia:

Bernardo Manzano Salaza

Centro de Hemodiálisis Montequinto

$\mathrm{Cl}$. Historiador Juan Manzano, 2

41089 Dos Hermanas (Sevilla)

berni.manzano@hotmail.com de forma planificada la Calidad, buscando como fin principal la satisfacción del paciente y buscamos mejoras continuas en todas las áreas posibles y así conseguir una satisfacción mayor de nuestros pacientes como objetivo final de nuestras acciones ${ }^{3}$.

Son muchas las actividades y registros que lleva Enfermería Nefrológica, lo cual nos llevó a plantear de alguna forma, un método que ayudara a controlar, medir, definir y mejorar las distintas funciones que estamos desarrollando; así podremos optimizar, planificar y personalizar los cuidados de Enfermería y el tratamiento que se aplica a los pacientes.

Nuestro Sistema de Gestión de Calidad en Enfermería está basado en la norma UNE-EN ISO 9001:2008, la cual va encaminada sobre todo a la satisfacción del cliente basado en el cumplimiento de unos requisitos específicos. En esta misma línea, queremos dejar claro que el modelo descrito está basado en la Gestión de la Calidad en el área de Enfermería, donde se vienen controlando distintos procesos e indicadores que, como veremos posteriormente, contribuyen a una mejora continua del Sistema de Gestión de la Calidad.

Como objetivo fundamental se planteó la participación de Enfermería en la aplicación del Sistema de Gestión de la Calidad adaptando y cumpliendo los requisitos y necesidades de nuestros pacientes, ofreciéndoles un servicio de tratamiento de hemodiálisis con los máximos niveles de Calidad, planteando como directrices principales: 
- Establecer Objetivos de Calidad e Indicadores de los mismos para los procesos existentes.

- Establecer por escrito las actividades que contribuyen a la Calidad y a la Protección, describiendo el modo de ejecutarlas y revisando el cumplimiento y eficacia de las actividades documentadas.

- Tener capacidad de respuesta en el servicio por Enfermería gracias al: trabajo en equipo, medición de resultados, diseños de acciones preventivas y correctivas, evaluación continua y planteo de objetivos con el fin de buscar la satisfacción de nuestros pacientes.

- Establecer una sistemática de medición de la satisfacción de pacientes y sus expectativas.

- Mantener y mejorar continuamente el Sistema de Gestión de la Calidad.
Presentamos un estudio descriptivo, observacional cuya población abarcó $64,72,70$, y 78 pacientes durante los años 2004, 2005, 2006 y 2007 respectivamente de nuestro centro de trabajo. Para ello se realizó un seguimiento y medición de un conjunto de INDICADORES ${ }^{4-7}$ (tabla 1), con el objeto de establecer qué se debe alcanzar y hasta qué punto se alcanzan determinados niveles.

Teniendo en cuenta los distintos indicadores anteriormente expuestos, los resultados obtenidos para cada uno ellos a lo largo de estos años de seguimiento y medición se reflejan en forma de gráfico (figuras 1-9).

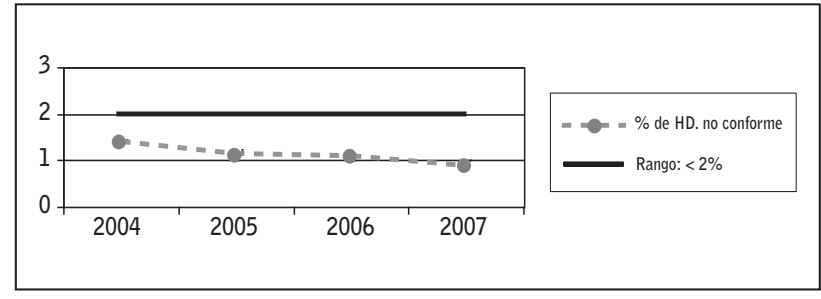

Figura 1: Fracción de diálisis no conforme

\begin{tabular}{|c|c|c|c|c|c|}
\hline Indicador & Valor Ref. & Fórmula Cálculo & Frecuencia & $\begin{array}{l}\text { Responsable } \\
\text { Medición }\end{array}$ & Registro \\
\hline \multirow{2}{*}{$\begin{array}{l}\text { Fracción de diálisis no } \\
\text { conforme }\end{array}$} & \multirow[t]{2}{*}{$<2 \%$} & $N^{\circ}$ hd no conformes & \multirow[t]{2}{*}{ Trimestral } & \multirow{2}{*}{$\begin{array}{l}\text { Jefe Unidad de } \\
\text { Diálisis }\end{array}$} & \multirow{2}{*}{$\begin{array}{l}\text { Informe } \\
\text { Trimestral }\end{array}$} \\
\hline & & $N^{0}$ sesiones $h d$ & & & \\
\hline \multirow{2}{*}{$\begin{array}{l}\% \text { pac. con Hto } \\
>30 \%\end{array}$} & \multirow[t]{2}{*}{$>90 \%$} & pacientes con $\mathrm{Hto} \geq 30 \%$ & \multirow[t]{2}{*}{ Mensual } & \multirow{2}{*}{$\begin{array}{l}\text { Nefrólogo } \\
\text { responsable } \\
\text { paciente }\end{array}$} & \multirow{2}{*}{$\begin{array}{l}\text { Informe } \\
\text { trimestral }\end{array}$} \\
\hline & & total pacientes trimestre & & & \\
\hline $\begin{array}{l}\% \text { pac. con kt/V } \\
>1,2\end{array}$ & $>80 \%$ & $\begin{array}{l}\text { pacientes con } \mathrm{kt} / \mathrm{vd}>1,2 \\
\text { total pacientes trimestre }\end{array} 100$ & Trimestral & $\begin{array}{l}\text { Nefrólogo } \\
\text { responsable } \\
\text { paciente }\end{array}$ & $\begin{array}{l}\text { Informe } \\
\text { trimestral }\end{array}$ \\
\hline \multirow{2}{*}{$\begin{array}{l}\text { \% pac. con Fe } \\
>100 \mathrm{ppb} .\end{array}$} & \multirow[t]{2}{*}{$>80 \%$} & pacientes con $\mathrm{Fe}>100 \mu \mathrm{g} / \mathrm{L}$ & \multirow[t]{2}{*}{ Trimestral } & \multirow{2}{*}{$\begin{array}{l}\text { Nefrólogo } \\
\text { responsable } \\
\text { paciente }\end{array}$} & \multirow{2}{*}{$\begin{array}{l}\text { Informe } \\
\text { trimestral }\end{array}$} \\
\hline & & total pacientes trimestre & & & \\
\hline \multirow{2}{*}{$\begin{array}{l}\text { \% pac. con P 2,5 y } \\
5,5 \mathrm{mg} / \mathrm{dl} \text {. }\end{array}$} & \multirow[t]{2}{*}{$>60 \%$} & pacientes con $P$ entre 2,5 y $5,5 \mathrm{mg} / \mathrm{dL} \times 100$ & \multirow[t]{2}{*}{ Bimensual } & \multirow{2}{*}{$\begin{array}{l}\text { Nefrólogo } \\
\text { responsable } \\
\text { paciente }\end{array}$} & \multirow{2}{*}{$\begin{array}{l}\text { Informe } \\
\text { trimestral }\end{array}$} \\
\hline & & total pacientes trimestre & & & \\
\hline Índice Mortalidad & $<14 \%$ & $\begin{array}{l}\text { Pacientes prevalentes fallecidos en hemodiálisis } \\
\text { sobre el total de pacientes que hayan estado } \\
\text { recibiendo dicha técnica en un periodo de un año } \\
(\%)\end{array}$ & Trimestral & $\begin{array}{c}\text { Jefe Unidad de } \\
\text { Diálisis }\end{array}$ & Informe Anual \\
\hline Índice Trasplantes & $>5 \%$ & $\begin{array}{l}\text { Pacientes prevalentes trasplantado sobre el total } \\
\text { de pacientes que hayan estado recibiendo dicha } \\
\text { técnica en un periodo de un año }(\%)\end{array}$ & Trimestral & $\begin{array}{l}\text { Jefe Unidad de } \\
\text { Diálisis }\end{array}$ & Informe Anual \\
\hline $\begin{array}{l}\text { Valoración } \\
\text { Satisfacción paciente }\end{array}$ & $\begin{array}{l}\geq 4 \\
\text { (sobre 5) }\end{array}$ & Según Procedimiento interno del centro & Anual & $\begin{array}{l}\text { Responsable de } \\
\text { Calidad }\end{array}$ & Informe Anual \\
\hline
\end{tabular}

Tabla 1: Indicadores de proceso 


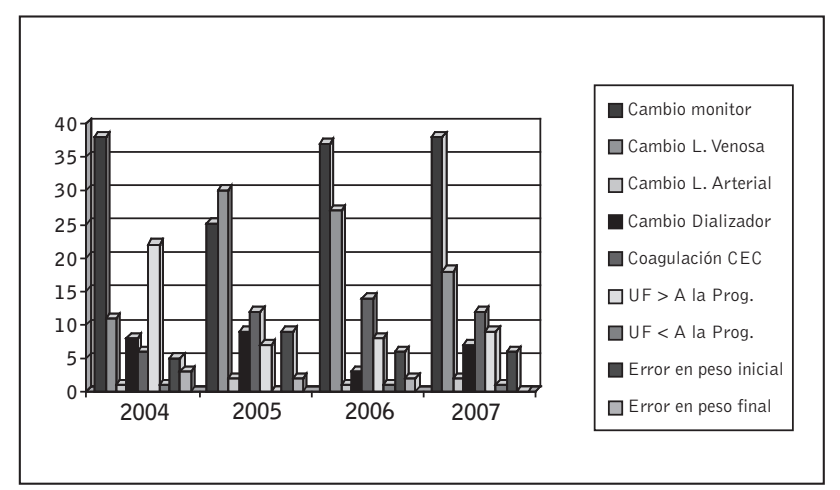

Figura 2: Distribución de las diálisis no conformes.

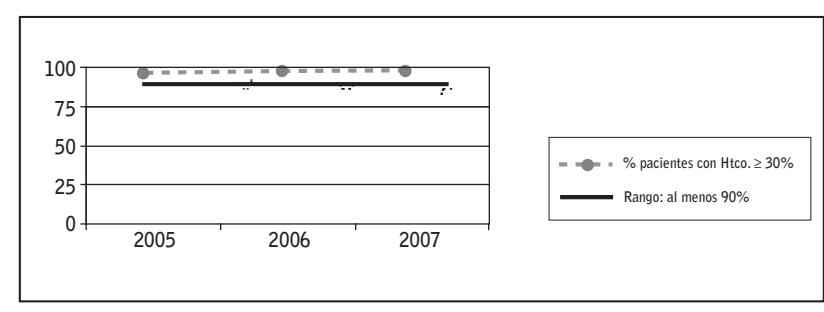

Figura 3: \% pacientes con hematocrito $\geq 30 \%$.

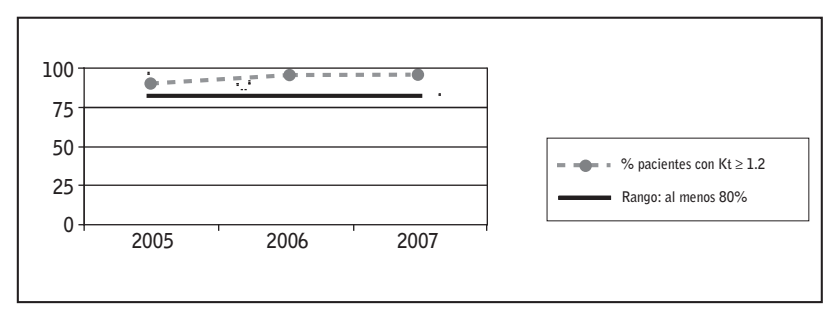

Figura 4: \% pacientes con $\mathrm{Kt} / \mathrm{V} \geq 1,2$.

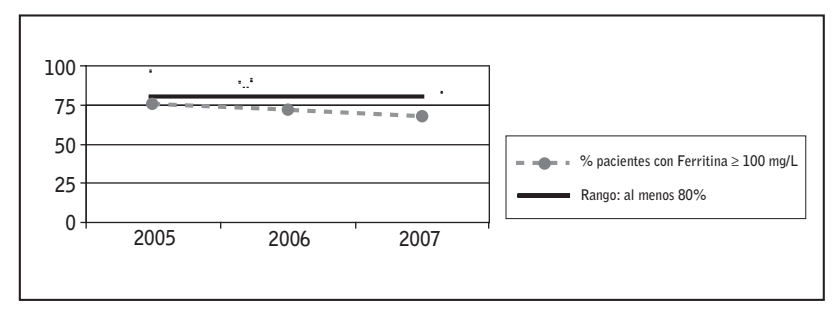

Figura 5: \% pacientes con Ferritina $\geq 100 \mathrm{mg} / \mathrm{L}$.

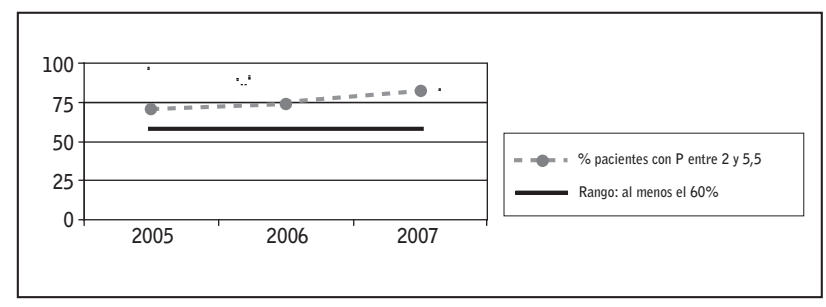

Figura 6: \% pacientes con niveles de fósforo entre 2,5 y 5,5 mg/dL.

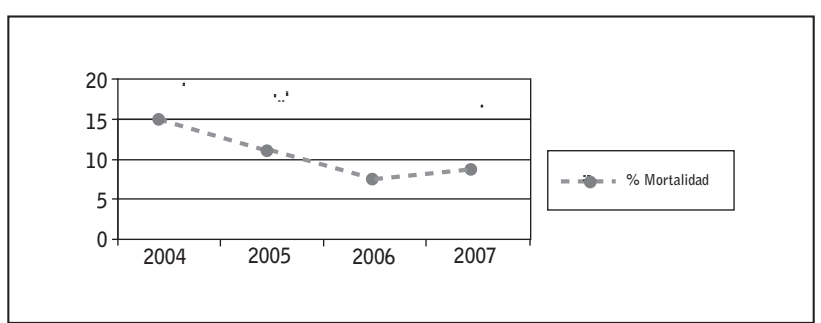

Figura 7: Índice de mortalidad.

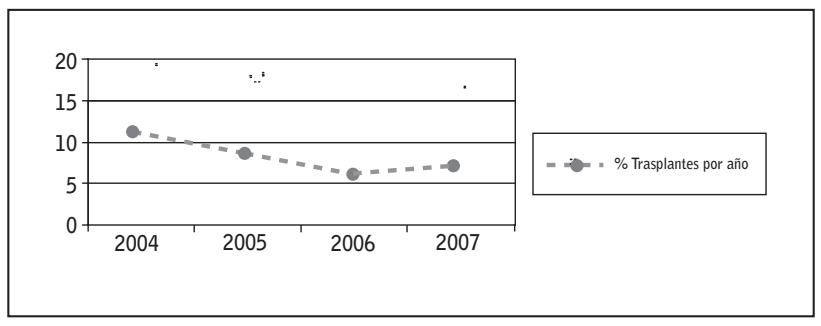

Figura 8: Índice de trasplantes.

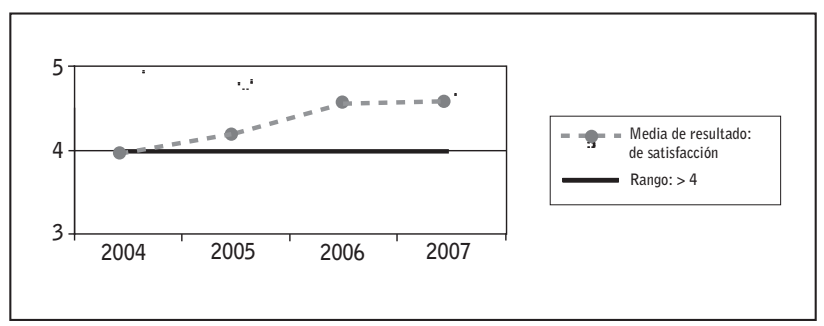

Figura 9: Valoración de satisfacción de los pacientes sobre el personal de enfermería.

El diseño de nuestro estudio nos permite plantear los distintos aspectos de la atención de Enfermería Nefrológica en lo que a Gestión de la Calidad se refiere, como profesionales de salud, con el fin de satisfacer las necesidades y expectativas de los pacientes con continua mejora. Es necesaria la calidad para la comprobación sistemática tanto de lo que se realiza como de los resultados que se producen o se esperan. Su aplicación disminuye la variabilidad de la práctica clínica e intenta buscar la eficiencia, esto es, la mejor relación posible entre los recursos consumidos y los resultados obtenidos ${ }^{8}$. Los resultados obtenidos expresan la necesidad de trabajar de una forma protocolizada con unas fases fundamentales: planificación, control y mejora continua. El contar con un Servicio de Calidad para el desarrollo de todas las cualidades y actitudes del personal de Enfermería, es beneficioso pudiendo participar todo el personal en numerosos indicadores decisivos, mostrando siempre una actitud positiva con la mejora y satisfacción del paciente. 


\section{Bibliografía}

1. Grupo de Trabajo sobre Gestión de Calidad de la Sociedad Española de Nefrología (SEN). Implantación de Sistemas de Gestión de Calidad en las unidades de Nefrología española. NEFROLOGÍA. 2006; 26 (2): 234-241.

2. AENOR. NORMA UNE-EN ISO 9001:2008. Sistemas de Gestión de la Calidad. Requisitos. Madrid; 2008.

3. La calidad de la acción de Enfermería. Enfermería Global. [Revista en Internet] ISSN 1695-6141. Revista electrónica semestral de Enfermería Nº 6 . http://www.um.es/eglobal/6/06d02.html.

4. Grupo de Trabajo sobre Gestión de Calidad de la Sociedad Española de Nefrología (SEN). Indicadores de calidad asistencial del proceso de hemodiálisis crónica para pacientes con insuficiencia renal crónica.; 2004. http: //www.senefro.org.

5. Grupo de Trabajo sobre Gestión de Calidad de la Sociedad Española de Nefrología (SEN). Estudio multicéntrico de validación de indicadores de calidad; 2004. http: //www.senefro.org.

6. Agencia de Calidad Sanitaria. Consejería de Salud. Manual de Estándares. Programa de Acreditación de unidades de Hemodiálisis; 2006.

7. Consejería de Salud. Junta de Andalucía. Tratamiento sustitutivo de la insuficiencia renal crónica: diálisis y trasplante renal: proceso asistencial integrado. Sevilla; 2005.

8. Rodolfo Crespo. Política de calidad en Hemodiálisis. Hospital Reina Sofía de Córdoba. www.revistaseden. org/files/art628_1.pdf. 